\title{
SECOND-ORDER BLIND IDENTIFIABILITY OF CERTAIN CLASSES OF MULTIPATH CHANNELS USING ANTENNA ARRAYS
}

\author{
V.U.Reddy $\quad$ C. B. Papadias ${ }^{2} \quad$ A. J. Paulraj ${ }^{2}$ \\ ${ }^{1}$ Indian Institute of Science, Bangalore 560 012, India \\ 2 Information Systems Laboratory, Stanford University, Stanford, CA 94305-4055, U.S.A.
}

\begin{abstract}
Recently, a number of classes of multipath channels which are not blindly identifiable from fractionally spaced samples and second-order cyclic spectra have been presented. In this paper, we consider the blind identification problem of these channels using multiple antennas and show that they will not in general give rise to any common roots among the sub-channels formed from the antennas, and hence, they can be identified from second-order statistics.
\end{abstract}

\section{INTRODUCTION}

Several recent methods on blind channel identification and equalization $[1,2,3,4,5,6]$ rely on a system model with multiple sub-channels driven by a single input. These methods depend on a condition that no common root exists among the sub-channel polynomials. In a recent letter, Ding [7] has pointed out several classes of multipath channels which are not identifiable from second-order cyclic spectra (SOCS). Most of these channels are band-limited (however the bandlimitedness property is not needed for the class of channels with delays equal to integer multiples of the symbol period). In arriving at the non-identifiability of these channels, Ding has assumed the sub-channels to arise from oversampling the output of a single sensor. We may also point out that although the band-limited channels are strictly unidentifiable from SOCS [8], several algorithms are recently proposed to identify these channels by estimating their finite duration impulse response approximations.

In this paper, we show that the classes of multipath channels [7] can be identified from second-order statistics (SOS) using multiple antennas. In particular, we show that the sub-channels formed from multiple antennas will not share any common root; if a common root arises, it can be eliminated by synchronized symbol rate sampling or oversampling.

\section{BACKGROUND}

Under a standard model for a linear digital modulation over a linear intersymbol interference channel, the received baseband signal at the receiver is represented as

$$
y(t)=\sum_{k} a_{k} h(t-k T)+v(t)
$$

where $a_{k}$ 's are the transmitted symbols, $T$ is the symbol period, $h(t)$ is the composite impulse response of the multipath channel and transmitter and receiver filters, and $v(t)$ is the additive channel noise which is assumed to be independent of $a_{k}$ 's. We assume that $h(t)$ has a finite duration.

\subsection{Oversampling a single sensor output}

Let the sampling interval be $T_{s}=T / P$ where $P$ is an integer. Define $y_{i}(k)=\left.y(t)\right|_{t=t_{0}+k T+(i-1) T / P}$ and similarly $h_{i}(k)$. We can then write

$$
y_{i}(k)=\sum_{l=0}^{L} a_{k-l} h_{i}(l)+v_{i}(k)
$$

where $L$ is an integer such that $h_{i}(l)=0$ for $l \geq L+1$ and for $l<0$ for $i=1,2, \ldots, P$ and $h_{i}(0) \neq 0$ and $h_{i}(L) \neq 0$ for some $i \in\{1,2, \ldots, P\}$.

\subsection{Outputs of multiple sensors}

We now assume an array of $M$ sensors. With some abuse of notation, let $y_{i}(t)$ and $h_{i}(t)$ denote the complex envelope of the signal received at the output of $i$-th sensor and the composite baseband impulse response of the channel from the transmitter to the output of $i$-th sensor, respectively. Then

$$
y_{i}(t)=\sum_{k} a_{k} h_{i}(t-k T)+v_{i}(t)
$$

Assuming symbol rate sampling, we obtain the same expression as (2) for $y_{i}(k)$. 


\subsection{Blind identifiability}

Let $H_{i}(z)=\sum_{l=0}^{L} h_{i}(l) z^{-l}$. The vector channel

$$
\mathbf{H}(z)=\left[H_{1}(z) H_{2}(z) \ldots H_{P}(z)\right]^{T}
$$

is identifiable up to a unitary scalar factor from the SOCS if and only if there is no complex $\rho$ such that $H_{i}(\rho)=0 \forall i \in\{1, \ldots, P\}$ (see[9]) (for the case of multiple sensors, $P$ should be replaced by $M$ ). An alternative statement of this common root condition is as follows. Consider the $P L$-length filter

$$
H\left(z_{1}\right)=\sum_{i=1}^{P} z_{1}^{-(i-1)} H_{i}\left(z_{1}^{P}\right)
$$

obtained by interleaving the impulse responses $h_{i}(k), i=$ $1,2, \ldots, P$. In (5), we have used $z_{1}$ instead of $z$ so as to emphasize that the coefficients of $H\left(z_{1}\right)$ are spaced at $T / P$ seconds apart while those of $H_{i}(z)$ are spaced at $T$ seconds. Then, the condition that $\left\{H_{i}(z)\right\}$ have a common root, say $\rho$, is equivalent to the condition that $H\left(z_{1}\right)$ has a set of $P$ zeros located symmetrically around a circle of radius $|\rho|^{1 / P}$ with origin as its center [9], which is the original form in which the commonroots condition was presented in [2].

One can use either of these conditions to examine the identifiability of the channels.

\section{IDENTIFIABILITY OF CERTAIN MULTIPATH CHANNELS}

Let $c(t)$ denote the continuous-time impulse response of a multipath channel (excluding that of transmitter and receiver filters). We can then express $c(t)$ as $c(t)=\sum_{q=0}^{Q-1} \alpha_{q} \delta\left(t-\tau_{q}\right)$ where $\alpha_{q}$ and $\tau_{q}$ are the fading coefficient and the propagation delay, respectively, of $q$-th path, $Q$ is the number of multipaths and $\delta(\cdot)$ is the Dirac delta function. Let $g(t)$ denote the effective continuous-time pulse shape which includes the effects of the transmitting and receiving filters. Then, the composite impulse response of the channel, $h(t)$, is given by $h(t)=c(t) * g(t)$ where $*$ denotes convolution. We assume that $g(t)$ has a finite duration.

\subsection{Multipath channels with delays equal to in- teger multiples of $T$ (Class I)}

These channels are described by

$$
c(t)=\sum_{q=0}^{Q-1} \alpha_{q} \delta(t-q T)
$$

It is shown in [9] that these channels give rise to common roots among the sub-channel polynomials obtained by oversampling, and hence, are not identifiable from SOCS (in [7], these channels are classified as Class I channels). We may point out here that though these channels are presented as band-limited channels, the band-limited assumption is not necessary for their nonidentifiability as is required for other classes of channels described in [7].

We now consider the sub-channels that we obtain from $M$ sensors. We assume that these sensors are omni-directional and they form a uniform linear array (this assumption is made for convenience of exposition only and the following conclusions hold for arbitrary arrays as well). Assuming that the $Q$ paths impinge the array from angles $\theta_{0}, \theta_{1}, \ldots, \theta_{Q-1}$ (measured with respect to the broadside direction of the array), the baseband equivalent impulse response of the channel between the transmitter and the output of $i$-th sensor, under the narrowband assumption of the transmitted signal, is given by

$$
\begin{aligned}
h_{i}(t) & =\left(\sum_{q=0}^{Q-1} \alpha_{q} e^{-j \frac{2 \pi d}{\lambda}(i-1) \sin \theta_{q}} \delta(t-q T)\right) * g(t) \\
& =\left(\sum_{q=0}^{Q-1} \alpha_{q} e^{-j \frac{2 \pi d}{\lambda}(i-1) \sin \theta_{q}} g(t-q T)\right)
\end{aligned}
$$

where $d$ is the inter-element spacing and $\lambda$ is the wavelength of the carrier frequency. The symbol-rate sampled response of the $i$-th sub-channel is then given by

$$
h_{i}(k)=\sum_{q=0}^{Q-1} \gamma_{i q} g(k-q), \quad \gamma_{i q}=\alpha_{q} e^{-j \frac{2 \pi d}{\lambda}(i-1) \sin \theta_{q}}
$$

where $h_{i}(k)=\left.h_{i}(t)\right|_{t=t_{0}+k T}$ and $g(k)=\left.g(t)\right|_{t=t_{0}+k T}$ Taking the z-transform, we get

$$
H_{i}(z)=\sum_{q=0}^{Q-1} \gamma_{i q} z^{-q} G(z)
$$

Note the difference between the characterization of $i$-th sub-channel obtained by oversampling [9] and that of (9). In the multiple sensors case, the common factor among the sub-channels comes from the pulse shape while in the oversampling case it comes from the multipath channel (see [9]). We now show how the common factor $G(z)$ can be eliminated by choosing a suitable pulse shape and synchronized symbol-rate sampling (or by using oversampling), thereby allowing the sub-channels to be identifiable from SOS. Let $g(t)$ be a Nyquist pulse (e.g. a pulse with raised cosine spectrum). This pulse has zero value at $t= \pm k T$ for $k \neq 0$. If the sampling of $y_{i}(t)$ is synchronized with the symbol timing, i.e., $t_{0}=0$, then $g(k)=1$ for $k=0$ and $g(k)=0$ for $k \neq 0$. This gives $G(z)=1$, and conse- 
quently, the sub-channel transfer function becomes

$$
H_{i}(z)=\sum_{q=0}^{Q-1} \gamma_{i q} z^{-q}
$$

Equation (10), combined with the definition of $\gamma_{i q}$ (see (8)), shows that the $M$ sub-channels will have no common roots as long as the arrival angles of the $Q$ multipaths are not the same or they do not correspond to array ambiguities.

Now suppose that synchronized symbol rate sampling is not possible, and instead, we oversample $h_{i}(t)$ by a factor $P$. We will then have

$$
h_{i p}(k)=\sum_{q=0}^{Q-1} \gamma_{i q} g_{p}(k-q), \quad p=1,2, \ldots, P
$$

where $h_{i p}(k)=\left.h_{i}(t)\right|_{t=t_{0}+k T+(p-1) T / P}$ and $g_{p}(k)=$ $\left.g(t)\right|_{t=t_{0}+k T+(p-1) T / P}$. The corresponding $z$-transfer function is given by

$$
H_{i p}(z)=\sum_{q=0}^{Q-1} \gamma_{i q} G_{p}(z) z^{-q}=G_{p}(z) \sum_{q=0}^{Q-1} \gamma_{i q} z^{-q}
$$

Note that $\left\{G_{p}(z)\right\}$ do not share in general any common factor since they correspond to $\mathrm{z}$-transforms of the sequences derived from different parts of the pulse shape, and the coefficients of the polynomial (second term in (12)) vary with antenna index $i$ as long as the multipath angles are not same or they do not correspond to array ambiguities. We may also remark here that according to (12), for a fixed $p$ we get a group of common roots among $\left\{H_{i p}(z)\right\}$, but these roots are not in general shared among the different $p$ 's. Thus, the $M P$ sub-channel polynomials do not share any common root, and hence, the vector channel

$\mathbf{H}(z)=\left[H_{11}(z) \ldots H_{1 P}(z) \ldots H_{M 1}(z) \ldots H_{M P}(z)\right]^{T}$

can be identified from SOS.

\subsection{Multipath channels with frequency nulls (Class II)}

In [7], Ding shows that multipath channels with frequency nulls in $[-\pi(1-\beta) / T, \pi(1-\beta) / T]$, where $\beta$ is the rolloff parameter, are not identifiable from SOCS. In particular, he shows that each frequency null in $[-\pi(1-\beta) / T, \pi(1-\beta) / T]$ gives rise to a set of $P$ roots in the oversampled response that are located uniformly around the unit circle. In arriving at this result, he implicitly assumes the overall channel frequency response (including that of transmitter and receiver filters) to be band-limited to $[-\pi(1+\beta) / T, \pi(1+\beta) / T]$; this bandlimitedness property is used in order to pick $P-1$ unit circle roots from the stop-band region of the composite channel response. Here, we show that for such multipath channels, the sub-channels formed from $M$ sensors will not suffer from common roots.

Consider the class of multipath channels with two paths [7]

$$
c(t)=\delta(t)+\delta\left(t-\frac{T}{(1-\eta)}\right), \quad \eta>\beta
$$

which has a frequency null at $\omega=\frac{\pi(1-\eta)}{T}$ or $\omega=$ $\frac{-\pi(1-\eta)}{T}$. Now consider the sub-channels obtained from the $M$ sensors of a uniform linear array. Following the steps similar to those used in arriving at (7), we obtain the impulse response of the $i$-th sub-channel as

$$
\begin{aligned}
& h_{i}(t)=e^{-j \frac{2 \pi d}{\lambda}(i-1) \sin \theta_{0}}(g(t)+g(t-\tau) \times \\
& \left.\times e^{-j \frac{2 \pi d}{\lambda}(i-1)\left(\sin \theta_{1}-\sin \theta_{0}\right)}\right), \quad \tau=T /(1-\eta)
\end{aligned}
$$

where $\theta_{0}$ and $\theta_{1}$ are the arrival angles of the direct and reflected paths, respectively. The symbol-rate sampled version of $h_{i}(t)$ can then be expressed as

$$
\begin{aligned}
h_{i}(k) & =e^{-j \frac{2 \pi d}{\lambda}(i-1) \sin \theta_{0}}\left(g(k)+\gamma_{i} g_{\tau}(k)\right) \\
\gamma_{i} & =e^{-j \frac{2 \pi d}{\lambda}(i-1)\left(\sin \theta_{1}-\sin \theta_{0}\right)}
\end{aligned}
$$

where $g_{\tau}(k)=\left.g(t-\tau)\right|_{t=t_{0}+k T}$. Taking the z-transform, we have

$$
\dot{H_{i}}(z)=e^{-j \frac{2 \pi d}{\lambda}(i-1) \sin \theta_{0}}\left(G(z)+\gamma_{i} G_{\tau}(z)\right)
$$

Observe that there is no common polynomial factor shared by the sub-channels. For $\tau=k T$, however, $G_{\tau}(z)=G(z) z^{-k}$ and it corresponds to a special case of the Class I channels.

To get more insight into the unit circle roots among $\left\{H_{i}(z)\right\}$, consider the following. Let $H(\omega)$ denote the Fourier transform of $h(t)$, and similarly $G(\omega)$, where $\omega$ is the analog radian frequency. The frequency response of the lowpass equivalent of the $i$-th sub-channel can be shown to be

$H_{i}(\omega)=\left(e^{-j \frac{2 \pi d}{\lambda}(i-1) \sin \theta_{0}}+e^{-j \frac{2 \pi d}{\lambda}(i-1) \sin \theta_{1}} e^{-j \frac{\omega T}{(1-\eta)}}\right) G(\omega)$

Since $G(\omega)$ is non-zero for $\omega \in(-\pi(1+\beta) / T, \pi(1+$ $\beta) / T$ ) (assuming a raised cosine pulse shape), the frequency null of $H_{i}(\omega)$ in $[-\pi(1-\beta) / T, \pi(1-\beta) / T]$, contributed by the term in the brackets, is different for each $i$. We may remark here that if we oversample $h_{i}(t)$ of (14) by a factor $P,\left\{H_{i p}(z)\right\}$ will contain a common unit circle root $\forall i$, but that root is not shared among 


\begin{tabular}{|r|l|}
\hline \hline $\begin{array}{c}\text { Common roots of the 4 } \\
\text { polynomials }(p=1)\end{array}$ & $\begin{array}{l}\text { Common roots of the 4 } \\
\text { polynomials }(p=2)\end{array}$ \\
\hline & \\
\hline-1.8497 & -0.6836 \\
\hline $6.0956 \pm 1.0206 j$ & $3.1926 \pm 2.2354 j$ \\
\hline $0.0493 \pm 3.7032 j$ & $-0.9604 \pm 2.9235 j$ \\
\hline$-0.1196 \pm 0.2861 j$ & $-0.0157 \pm 0.2936 j$ \\
\hline $0.1983 \pm 0.1476 j$ & $0.1832 \pm 0.0794 j$ \\
\hline
\end{tabular}

Table 1: Numerical results

different $i$ 's as long as the multipath angles are not same or they do not correspond to array ambiguities. We arrive at similar conclusions in respect of the other classes described in [7].

Thus, the classes of multipath channels discussed above do not suffer from identifiability based on SOS if we use multiple antennas in place of oversampling.

\section{NUMERICAL EXAMPLES}

We consider a 2-path model for a channel under Class I with $\theta_{0}=0^{\circ}, \theta_{1}=30^{\circ}, \alpha_{1}=1, \alpha_{2}=0.5$, and a raised cosine pulse with $\beta=0.3$. The two paths are separated by one symbol period $T$ in time. We assumed the pulse to be limited to \pm 5 baud intervals. Note that oversampling in this case will result in a common root, making the channel non-identifiable from SOCS. We consider a uniform linear array with $M=4$ and $d / \lambda=0.5$. In the case of zero offset $\left(t_{0}=0\right), H_{i}(z)=1+0.5 e^{-j \pi(i-1) / 2} z^{-1}$. Each of these polynomials has a single root equal to $-0.5,0.5 j$, 0.5 and $-0.5 j$, respectively, resulting in no common roots, thereby making SOS-based identifiability possible. We then choose a non-zero offset $t_{0}=0.2 T$. Now, the degree of each sub-channel polynomial is ten. As predicted by (9), the four polynomials have nine common roots and the 4 roots mentioned above will be distributed (one each) among these polynomials to form the tenth root. If we consider oversampling with $P=2$ (see (12)), the four polynomials corresponding to $p=2$ will share again nine common roots which are, however, different from those corresponding to $p=1$, as shown in the following table. Once again, the 4 roots mentioned above will be distributed (one each) among the 4 polynomials corresponding to $\forall i$. Thus, these results corroborate our predictions.

\section{CONCLUSIONS}

In this paper, we have considered the classes of multipath channels [7] that are shown to be unidentifiable from SOCS . Using multiple antennas and oversampling (or synchronized symbol rate sampling), we have shown that the sub-channels so obtained do not suffer from common roots, and hence, these multipath channels can be identified from SOS.

\section{REFERENCES}

[1] L. Tong, G. Xu, and T. Kailath. "A new approach to blind identification and equalization of multipath channels". In Proc. 25 th Asilomar Conference on Signals, Systems, and Computers, pages 856-860, Pacific Grove, CA, 1991.

[2] L. Tong, G. Xu, and T. Kailath. "Blind identification and equalization of multipath channels: a time domain approach ". IEEE Trans. on Information Theory, 40(2):340-349, March 1994.

[3] Z. Ding and Y. Li. "On channel identification based on second-order cyclic spectra". IEEE Trans. Signal Processing, 42:1260-1264, May 1994.

[4] D. T. M. Slock. "Blind fractionally-spaced equalization, perfect-reconstruction filter banks and multichannel linear prediction". In Proc. ICASSP 94 Conference, pages IV-585-IV-588, Adelaide, Australia, April 1994.

[5] D. T. M. Slock and C. B. Papadias. "Further results on blind identification and equalization of multiple FIR channels". In Proc. ICASSP 95 Conference, volume 4, pages 1964-1967, Detroit, Michigan, May 1995.

[6] E. Moulines, P. Duhamel, J. F. Cardoso, and S. Mayrargue. "Subspace methods for the blind identification of multichannel FIR filters". In Proc. ICASSP 94 Conference, pages IV-573-IV576, Adelaide, Australia, 1994.

[7] Z. Ding. "Characteristics of band-limited channels unidentifiable from second-order cyclostationary statistics". IEEE Signal Processing Letters, 3(5):150-152, May 1996.

[8] Y. Chen and C. L. Nikias. "Identifiability of a bandlimited system from its cyclostationary output autocorrelation". IEEE Trans. on Signal Processing, 42:483-485, Feb. 1994

[9] J. K. Tugnait. "On blind identifiability of multipath channels using fractional sampling and secondorder cyclostationary statistics". IEEE Trans. Information Theory, 41:308-311, Jan. 1995. 